\title{
A Distributed Asynchronous Directional-to-Directional MAC Protocol for Wireless Ad Hoc Networks
}

\author{
Emad Shihab, Student Member, IEEE, Lin Cai, Member, IEEE, and Jianping Pan, Senior Member, IEEE
}

\begin{abstract}
The use of directional antennas in ad hoc networks has received growing attention because of its high spatial reuse and high antenna gains. The medium access control (MAC) protocol design with directional antennas is nontrivial due to the deafness and collision problems. The existing protocols assumed that the nodes can operate in both directional and omnidirectional modes. However, using both modes leads to the asymmetry-in-gain problem. In this paper, we propose a directional-to-directional (DtD) MAC protocol, where both senders and receivers operate in a directional-only mode. We also derive the saturation throughput of ad hoc networks using DtD MAC. The analytical and simulation results show that DtD MAC can significantly improve the throughput and maintain good fairness among competing flows. We further evaluate the DtD MAC with millimeter-wave (mmWave) communication technologies, which rely on a directional antenna to perform well and can achieve gigabit data rates. The results obtained are exciting and show that DtD MAC is a promising candidate for mmWave ad hoc networks.
\end{abstract}

Index Terms-Ad hoc networks, directional antennas, medium access control (MAC) protocols, millimeter wave (mmWave).

\section{INTRODUCTION}

$\mathbf{T}$ HE USE of directional antennas in ad hoc networks has received growing attention [2] thanks to the higher antenna gain, better spatial reuse, longer transmission range, and lower interference between directional antennas. In addition, new millimeter-wave (mmWave) communication technologies have emerged for gigabit wireless personal area networks [3]. Millimeter-wave operates in the license-free frequency band from 57 to $64 \mathrm{GHz}$ [4], and its emission power limit imposed by the Federal Communications Commission is much less restrictive than that for ultrawideband communications. However, at $60 \mathrm{GHz}$, the signals suffer from high path loss due to oxygen absorption and atmospheric attenuation. Thus, a directional antenna is normally used by mmWave systems to combat the high path loss [5].

Manuscript received October 3, 2008; revised April 12, 2009. First published June 2, 2009; current version published November 11, 2009. This work was supported in part by grants from the Natural Sciences and Engineering Research Council of Canada. This paper was presented in part at the IEEE Global Telecommunications Conference, New Orleans, LA, November 30December 4, 2008. The review of this paper was coordinated by Dr. J. Deng.

E. Shihab is with the School of Computing, Queen's University, Kingston, ON K7L 3N6, Canada (e-mail: emads@cs.queensu.ca).

L. Cai is with the Department of Electrical and Computer Engineering, University of Victoria, BC V8W 3P6, Canada (e-mail: cai@uvic.ca).

J. Pan is with the Department of Computer Science, University of Victoria, BC V8W 3P6, Canada (e-mail: pan@uvic.ca).

Digital Object Identifier 10.1109/TVT.2009.2024085
Using directional antennas in ad hoc networks imposes new challenges for medium access control (MAC) protocols as the nodes do not normally have complete knowledge about their neighborhood, which results in deafness and collision problems. There have been a number of MAC protocols [6]-[9] specifically designed to work with directional antennas. Most of them are based on the IEEE 802.11 distributed coordination function (DCF) MAC protocol and use different flavors of the request-to-send and clear-to-send (RTS/CTS) handshake procedure to cope with the challenges introduced by directional antennas. In addition, the directional network-allocation vector (DNAV) and the Angle-of-Arrival (AoA) caching are used for neighbor discovery [10]; with DNAV, nodes keep a record of the ongoing transmissions by their neighbors in each direction, and AoA caching keeps the angles of any message that a node overhears.

To the best of our knowledge, most existing MAC protocols for ad hoc networks with directional antennas require that nodes be able to operate in both directional and omnidirectional modes. Typically, the omnidirectional mode is used by idle nodes to capture directional RTS (DRTS) messages that may be sent by their neighbors. This alleviates the deafness problem defined in [11] with a higher cost of equipping each node with two types of antennas. Furthermore, operating in both modes may defeat the purpose of using directional antennas due to the asymmetry-in-gain problem [12], for instance, node $S$ 's omnidirectional transmission may not reach node $R$, but node $R$ is within node $S$ 's directional transmission range. The asymmetric-in-gain problem magnifies the deafness problem if the control messages are sent omnidirectionally with a lower transmission range or idle nodes sense omnidirectionally with a lower sensing range. In [1], it was concluded that the omnidirectional transmitting and receiving of control packets will in fact impede the ability of directional antennas to achieve a higher network throughput.

To overcome the aforementioned problems, we are prompted to equip nodes with a single directional antenna, which are either switched beam or steerable. However, using directionalonly antennas introduces great challenges at the MAC layer. For example, the deafness problem is magnified. As nodes can only sense one direction at any given time instance, it has less of a chance to properly set its DNAV for all ongoing communications. Dealing with these problems requires the use of a new efficient wireless MAC protocol that can handle the new challenges imposed by directional-only antennas in ad 
hoc networks with no central coordination. In this paper, we propose a wireless MAC protocol called the directional-todirectional (DtD) MAC protocol that is specifically designed for nodes with directional-only antennas in ad hoc networks.

The main contributions of this paper are threefold. First, we propose a new wireless MAC protocol, i.e., DtD MAC, for DtD transmissions, which is built on the requirement that nodes are equipped with a single directional antenna. The proposed protocol is fully distributed, does not require synchronization, eliminates the asymmetry-in-gain problem evident in other directional MAC protocols (e.g., [7]), and alleviates the effect of deafness and collisions. Second, the performance of DtD MAC is studied in terms of saturation throughput and fairness metrics. To achieve this, we build an analytical model to capture the behavior of DtD MAC and verify the results with extensive simulations. Finally, to gain insights into the performance of DtD MAC for very high data rate technologies, we use the proposed analytical model to study the performance of DtD MAC for the emerging mmWave technology, and the results are promising.

The rest of this paper is organized as follows: Section II briefly describes the proposed DtD MAC protocol. The analytical model used to study the performance of DtD MAC is detailed in Section III. Section IV evaluates the performance of DtD MAC with extensive simulations. The related work is discussed in Section V, followed by the concluding remarks and further research issues in Section VI.

\section{DtD MAC PROTOCOL}

In this section, we outline the structure of the DtD MAC protocol. A shorter version describing DtD MAC can be found in [20], and a detailed description of DtD MAC can be found in [13]. Here, we still provide the basic explanation for the components used in the protocol to ensure that readers have sufficient background information to understand the DtD MAC protocol model and analysis presented in this paper. For brevity of presentation, we assume that switched-beam antennas with $M$ sectors are used in ad hoc networks.

In a nutshell, sending nodes in the DtD MAC cache the location information about their neighbor nodes. This information is later used to determine the direction in which it should first try to send DRTS messages. Idle nodes (potential receivers) continuously scan through their antenna sectors to emulate omnidirectional antennas. When they hear a DRTS intended for themselves, they lock in the respective direction and respond with a directional CTS (DCTS). Nodes that overhear ongoing communications accordingly set their DNAVs to refrain from interrupting the ongoing communications in these directions.

The next few sections describe the mechanisms used to support the DtD MAC operation.

\section{A. DNAV}

The network-allocation vector (NAV) is used in the IEEE 802.11 MAC protocol. Each node maintains a NAV that is updated from the duration field of the overheard RTS/CTS packets. For the case of directional antennas, the use of a

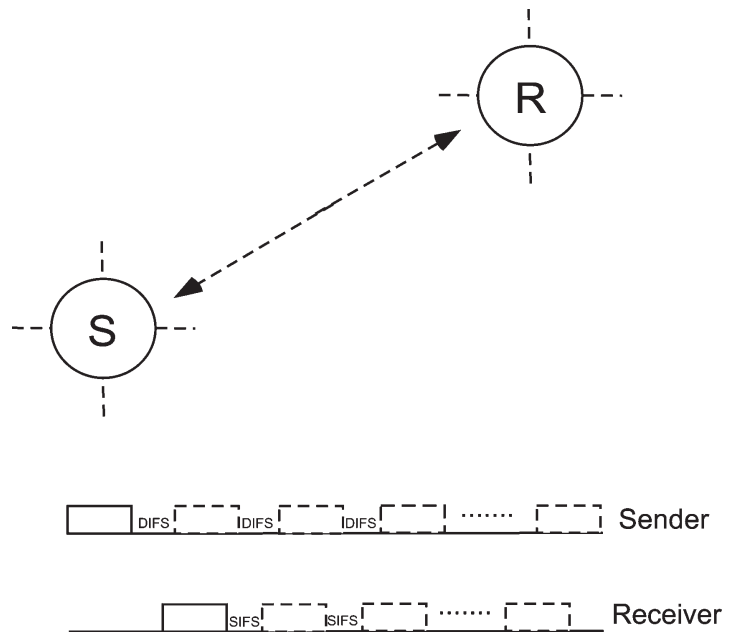

Fig. 1. Handshake procedures for directional-only antennas.

similar mechanism called DNAV was proposed in [8] and [10]: a node keeps a NAV for each direction or sector. The DtD MAC protocol also uses this DNAV mechanism.

\section{B. AoA Caching}

To improve the efficiency of the MAC protocol, senders need to estimate the direction of the intended receiving node. To achieve this, each node estimates and caches the AoA of any messages it overhears. This AoA caching mechanism was first introduced in [8]. The AoA information is updated when a node hears a new message from its neighbors. Before sensing the medium, a sending node checks its AoA cache to determine the receiver's most likely direction.

\section{Continuous Sector Scanning by Idle Nodes}

To minimize the effect of directional deafness, having idle nodes continuously switch their sensing directions in a clockwise (or anticlockwise) manner is the key. In essence, such a behavior emulates the presence of an omnidirectional antenna at the receiver. The idle nodes shall spend DRTS + SIFS $+\chi_{\mathrm{BO}}$ time in each sector. $\chi_{\mathrm{BO}}$ is added to compensate for the time that a sending node may spend in the backoff (BO) process.

When a node hears a transmission on one of its sectors, it sets its DNAV and continues to sequentially scan through all the other sectors. This approach has two main advantages: 1) It would reduce the deafness problem by reducing the chance that the receiver points to another direction and cannot be reached by a sender, and 2) more nodes properly setting their DNAV can alleviate the directional hidden-terminal problem.

However, this gain comes with an increased number of handshake messages that need to be sent by the sender, as demonstrated by the following example. Consider the scenario shown in Fig. 1. Assume that a sending node $S$ would like to engage in communication with a receiving node $R$. Further assume that these two nodes are not synchronized. If node $R$ was idle and continuously switching between beams, then node $S$ would be required to send multiple DRTS messages before it could successfully initiate communication with node $R$. The exact number of DRTS messages that node $S$ is required to send 
depends on the $\mathrm{BO}$ algorithm used and the number of antenna sectors $M$, which will be explained below.

\section{DRTS/DCTS/DATA/ACK Handshake}

In ad hoc networks, MAC protocols use RTS/CTS messages to mitigate the hidden-terminal problem. With directional antennas, when the nodes are not synchronized, a sender may change its direction and attempt to send in a direction that is already in use (e.g., another pair of nodes is already communicating). To solve this problem, a sender should sense the medium for a sufficiently long period of time before sending its DRTS messages. This sensing period includes the transmission time of a DATA packet plus an SIFS period. This way, a sender will always overhear either an ACK or DATA packet of the ongoing transmission taking place in a certain direction and refrain from transmitting to avoid collisions.

In addition, to guarantee that the sender captures the receiver, it has to send multiple DRTS messages in each direction. In our protocol, the sender will check the AoA cache to estimate the direction of the receiver. When the DNAV for that direction is not blocked (i.e., the medium is free), it senses that the medium is idle for DATA + SIFS time and sends up to $2 M$ DRTS messages to the receiver. When no DCTS is received, the sender will invalidate the AoA cache entry. When the direction of the receiver is unknown, a sender randomly chooses a new direction not being blocked by the DNAV and transmits up to $2 M$ DRTS messages in that direction. In the worst case, a sender would have to send $2 M$ messages in $M$ directions for each retry. This would cause the sender to send up to $2 M^{2}$ DRTS messages. Although the DRTS/DCTS messages still cannot totally eliminate the hidden-terminal problem, multiple small DRTS/DCTS messages and extended channel-sensing periods can reduce the occurrence of such problems for ad hoc networks with directional-only antennas.

When the receiver successfully captures the DRTS, it caches the sender's AoA, responds with a DCTS, locks its antenna in the direction of the sender, and waits for DATA packets. Upon receiving a DATA packet that is intended for itself, the receiver replies with an ACK. When a sending node does not receive an ACK within an $\mathrm{ACK}_{\text {TIMEOUT }}$ time, it backs off and retries until it reaches the retry limit. If the DATA is not received within

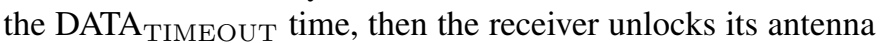
and continues sector scanning.

\section{E. BO Scheme}

In omnidirectional MAC protocols, when the receiver does not reply with a CTS, a sender should $\mathrm{BO}$ a random period before retry, and the average BO time is exponentially increased after each failed attempt. This is because the unanswered RTS messages are most likely due to collisions, and increasing the BO time can reduce the probability of collision. However, when using directional antennas, the deafness problem is introduced, and most of the unanswered DRTS messages may be due to deafness, since a directional receiving node may only sense one direction at any given time. Therefore, the binary exponential BO algorithm used in the IEEE 802.11 MAC protocol may not be efficient in the DtD MAC protocol. Since a sender is required to send up to $2 M$ DRTS messages in each direction, it may be required to increase its $\mathrm{BO}$ window up to $2 M$ times during this stage. To ensure that a sending node can capture its intended receiver with at most $2 M$ DRTS attempts and to alleviate the effect of deafness, we propose the following BO scheme: For the $(2 i-1)$ th DRTS message $(i=1,2, \ldots, M)$, the contention window size $W_{2 i-1}$ is randomly chosen from $\left[0, W_{\max }\right)$, and for the $2 i$ th DRTS message, $W_{2 i}$ is randomly chosen from $\left[W_{\max }-\right.$ DRTS - SIFS $\left.-W_{2 i-1}, W_{\max }\right)$. This is to ensure that an idle receiver can capture a DRTS message no matter which direction it begins to sense, as explained below.

First, without synchronization, an idle node should spend at least DRTS + SIFS $+\mathrm{BO}_{\max }$ in each direction to ensure that if there are DRTS messages coming from that direction, the idle node can capture at least one of them. Second, in the worst case, the idle node will spend $(M-1)(\mathrm{DRTS}+$ $\left.\mathrm{SIFS}+\mathrm{BO}_{\max }\right)$ time in other directions before it senses the sender's direction, and therefore, the sender should ensure that the duration between the beginning of the first DRTS to the beginning of the $2 M$ th DRTS is longer than $(M-1)($ DRTS + $\left.\mathrm{SIFS}+\mathrm{BO}_{\max }\right)$, i.e.,

$$
\begin{aligned}
(2 M-1)(\mathrm{DRTS} & + \text { SIFS })+\sum_{i=2}^{2 M} \mathrm{BO}_{i} \\
& \geq(M-1)\left(\mathrm{DRTS}+\mathrm{SIFS}+\mathrm{BO}_{\max }\right)
\end{aligned}
$$

where $\mathrm{BO}_{i}$ is the $\mathrm{BO}$ time before the $i$ th DRTS message. To ensure (1), a sufficient condition is

$$
\mathrm{BO}_{2 i-1}+\mathrm{BO}_{2 i} \geq \mathrm{BO}_{\max }-\text { DRTS }- \text { SIFS }
$$

for $i=1,2, \ldots, M$.

If $\mathrm{BO}_{2 i-1} \in\left[0, \mathrm{BO}_{\max }\right)$, then choosing $\mathrm{BO}_{2 i}$ from $\left[\mathrm{BO}_{\max }-\mathrm{DRTS}-\mathrm{SIFS}-\mathrm{BO}_{i}, \mathrm{BO}_{\max }\right)$ will ensure that (2) holds. Therefore, our BO scheme can ensure an idle receiver to capture at least one DRTS from the sender. The key parameter in the BO scheme is $W_{\max }$, which should appropriately be chosen to balance the time wasted during unnecessary $\mathrm{BO}$ and the time wasted due to transmission collision.

\section{F. Control Flow of the DtD MAC Protocol}

Fig. 2 outlines the normal operation of the DtD MAC protocol. As specified, a sender only attempts to send after it senses the medium, in the direction of transmission, idle for DATA + SIFS and when that direction is not blocked by DNAV. When the direction of the receiver is unknown, the sender sends the DRTS in a randomly chosen direction. In both cases, at most $2 M$ DRTS messages are sent in any direction. After sending the DRTS, it waits in the same direction for the DCTS. If the DCTS is not received, then the sender should BO and send the DRTS again. If the DCTS is received, then the node sends the DATA and waits for the ACK.

\section{G. Design Features of the DtD MAC Protocol}

The main features and advantages of the proposed DtD MAC protocol are summarized as follows. 


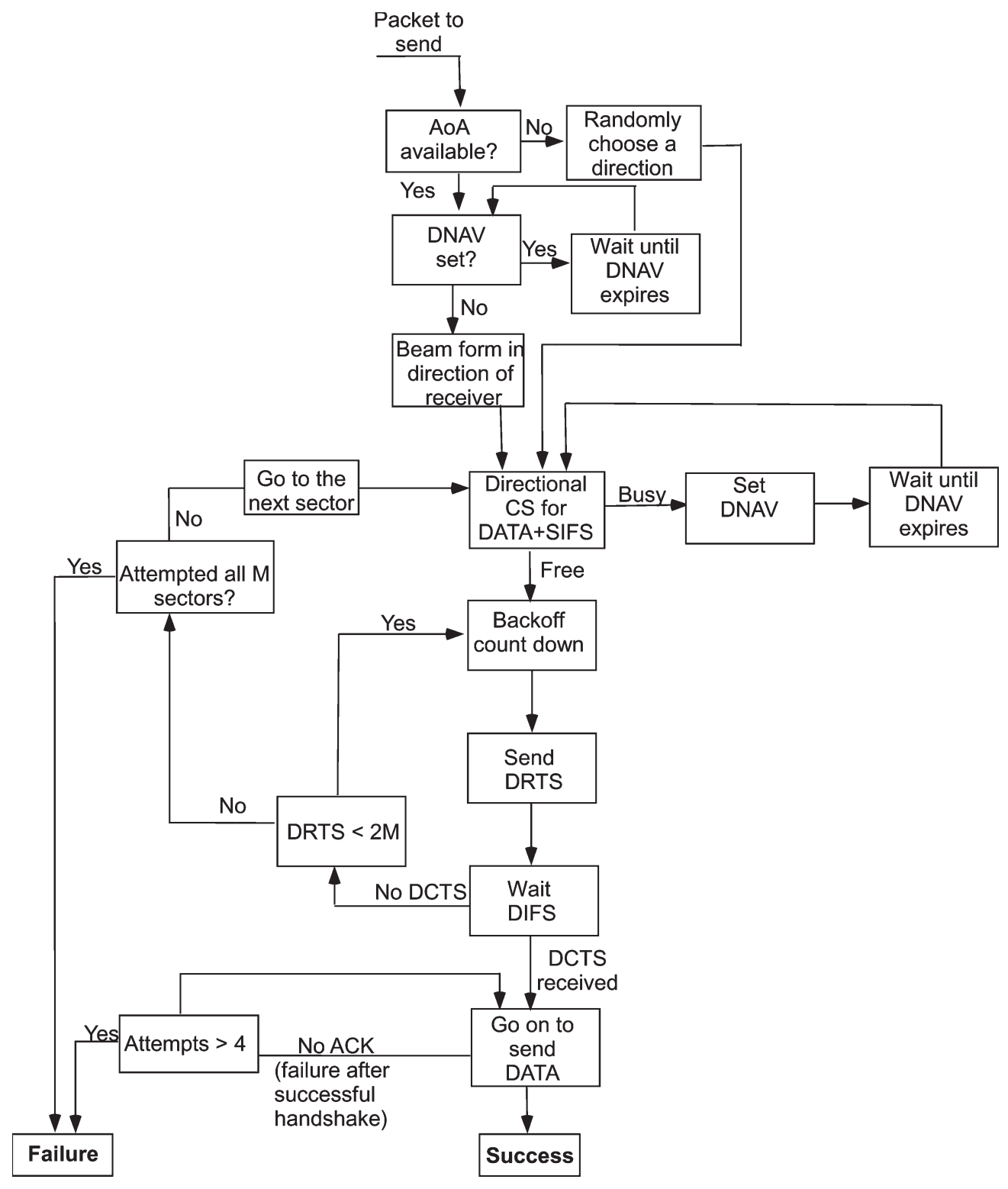

Fig. 2. Control flow of the DtD MAC protocol.

First, since DtD MAC only relies on directional antennas, we can effectively eliminate the asymmetry-in-gain problem. Second, the DtD MAC protocol requires no centralized controller and can operate in a fully distributed manner. This is an advantage unmatched in existing protocols exclusively using directional antennas. Being fully distributed makes the protocol more feasible and scalable for implementation. Third, sending multiple DRTS messages in each direction allows the network to operate without synchronization, as synchronization is difficult to achieve in ad hoc networks. In addition, since a sender is required to send multiple DRTS messages in each direction, it minimizes the deafness problem. Each sender is required to sense the medium for DATA + SIFS time in a new direction before transmitting. This reduces the chance for collisions due to the deafness problem in the DtD MAC protocol.

These advantages highlight the fact that DtD MAC is practical in ad hoc networks. In the next section, we analytically study the performance of the DtD MAC protocol. The sat- uration throughput is obtained, given the number of channel contenders, the packet size, and the antenna beamwidth.

\section{Analytical Model of the DtD MAC Protocol}

In this section, we first introduce the antenna model and then use it as a basis to study the network saturation throughput.

\section{A. Antenna Model}

The two types of practical directional antennas are phasedarray antennas and switched-beam antennas. Phased-array antennas achieve beam steering by constantly changing the phase of the antenna elements that constitute the array. Switchedbeam antennas are equipped with a number of directional antenna elements oriented in some predefined directions [14]. They can electronically switch between beams, thus exhibiting some degree of steering. Switched-beam antennas are 
more cost effective than phased-array antennas [15]. However, their limited beam-steering capability may make the transmitter-receiver beam alignment pretty rigid.

In this paper, we assume a low-cost switched-beam antenna at each node that consists of $M$ sectors, where $M=2 \pi / \theta$. In our study, $\theta$ varies from $\pi / 6$ to $\pi$ for different $M$. We assume that a node can either directionally transmit or receive at any given time instance, but not both. In all cases, all nodes use directional antennas with identical fixed beamwidth.

To consider the physical gain of using directional antennas, the receiver power is based on the following model:

$$
P_{R}=P_{T} \times G_{T} \times G_{R} \times G_{C}
$$

where $P_{T}, G_{T}, G_{R}$, and $G_{C}$ denote the transmission power, transmitter antenna gain, receiver antenna gain, and channel gain, respectively. At the transmitter, we adjust the achievable data rate according to $M$. Using Shannon's channel capacity equation, we can derive the achievable data rate using directional antennas (denoted with subscript $D$ ) and omnidirectional antennas (denoted with subscript $O$ ) as $C_{O}=$ $W \log _{2}\left(\mathrm{SNR}_{O}+1\right)$ and $C_{D}=W \log _{2}\left(\mathrm{SNR}_{D}+1\right)$, respectively, where $W$ is the signal bandwidth.

Assuming $G_{T}=G_{R}$, we obtain

$$
k_{M}=\frac{C_{D}}{C_{O}} \approx \frac{\log _{2}\left(G_{D}^{2}\right)+\log _{2}\left(\mathrm{SNR}_{O}\right)}{\log _{2}\left(G_{O}^{2}\right)+\log _{2}\left(\mathrm{SNR}_{O}\right)} .
$$

Since the amount of total RF energy remains the same, with more antenna sectors, the area of the distributed RF energy is smaller, which results in a higher antenna gain [16]. Assuming $G_{O}$ to be unity, $G_{D}$ to be proportional to $M$, and the SNR to be much larger than $1, k_{M}$ can be approximated by

$$
k_{M} \approx \frac{2 \log _{2} M+\log _{2}\left(\mathrm{SNR}_{O}\right)}{\log _{2}\left(\mathrm{SNR}_{O}\right)}
$$

and the data rate for a node with $M$ antenna sectors is

$$
\text { DataRate }_{M}=k_{M} \times \text { DataRate }_{O}
$$

where DataRate $_{O}$ is the achievable data rate for omnidirectional antennas.

\section{B. Network Throughput Analysis}

In this section, we study the system's MAC layer saturation throughput. This performance metric indicates the maximum throughput that can be achieved in all scenarios, assuming that all nodes in the network are continuously loaded with packets for transmission.

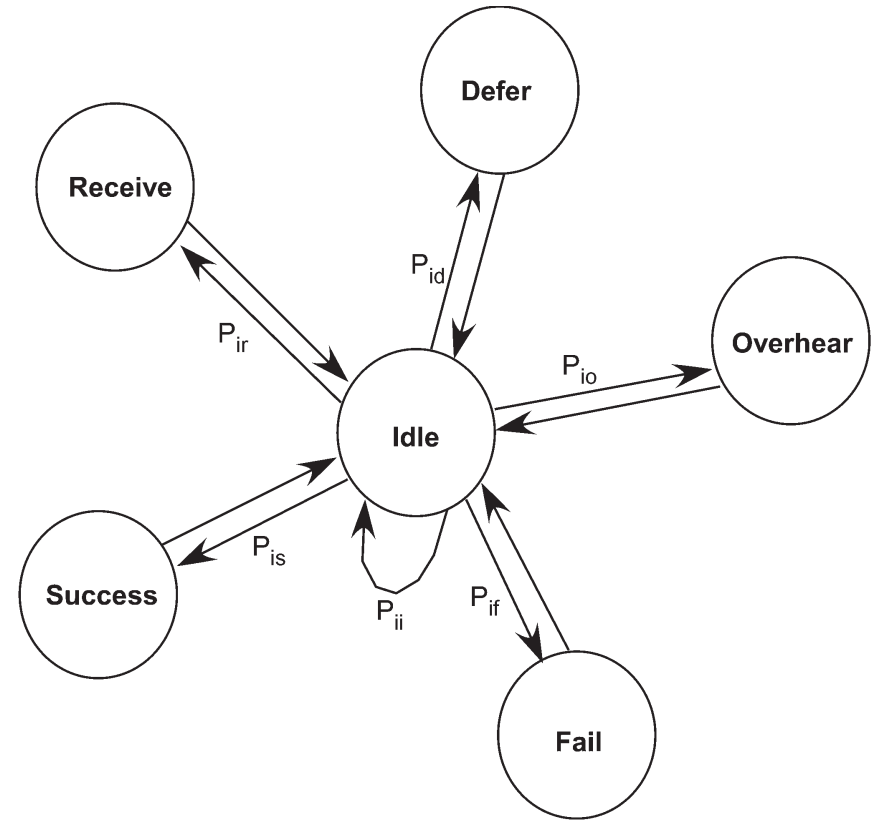

Fig. 3. Markov chain state transition diagram for each node.

Consider a system with $N$ active nodes uniformly distributed in the field. Each node is equipped with an $M$-sector antenna, and each source node randomly picks a destination node. Each node can be in one of six states. The state transition process of a node is represented by a discrete-time Markov chain model shown in Fig. 3. When in the idle state, a node is backing off, and the channel is observed to be idle. The success state and the failure state are the states at which a node resides after completing a successful packet transmission or having a failed transmission, respectively. The receive state is the state at which a node successfully receives a packet. The defer state is the state at which a node enters when it has a packet to send but is forced to defer its transmission due to an entry in its DNAV for the intended direction. The overhear state denotes the state where a node overhears others.

Denote $\tau$ as the packet transmission probability and $p$ as the failure probability of a transmission. The random $\mathrm{BO}$ scheme used in the DtD MAC protocol is shown in Fig. 4. Using an approach similar to that in [17], we note that

$$
b_{i-1,0} \times p=b_{i, 0} \rightarrow b_{i, 0}=p^{i} \times b_{1,0}, \quad 1<i<2 M .
$$

Owing to the regularities in the Markov chain, for each $k \in\left[0, W_{\max }-1\right], b_{i, k}$ is given in (8), shown at the bottom of the page.

$$
b_{i, k}= \begin{cases}\frac{W_{\max }-k}{W_{\max }}\left(\sum_{j=1}^{2 M} b_{j, 0}\right)(1-p), & i=1, \\ \frac{p \times b_{i-1,0}}{W_{\max }}\left[\sum_{W_{i-1}=0}^{W_{\max }-1} \sum_{k=W_{\max }-1-C-W_{i-1}}^{W_{\max }-1}\left(1-\frac{k}{1+W_{i-1}+C}\right)\right], & i>1 \text { and is even, } \\ \frac{p \times b_{i-1,0}\left(W_{\max }-k\right)}{W_{\max }}, & i>1 \text { and is odd }\end{cases}
$$




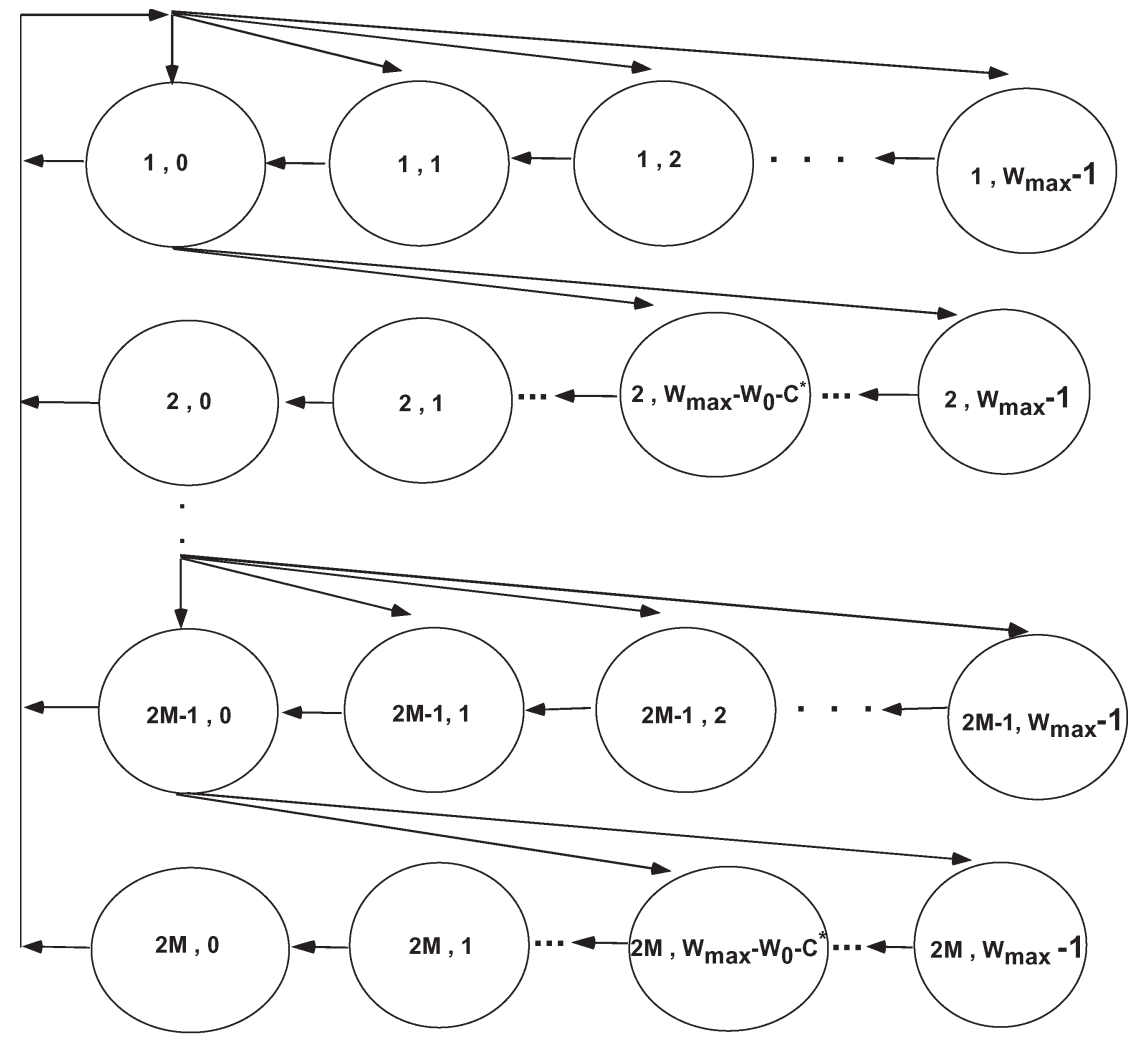

$C^{*}=$ DRTS + SIFS number of slots

Fig. 4. Random BO mechanism used in the DtD MAC protocol.

By imposing the normalization condition and using $\sum_{j=1}^{2 M} b_{j, 0}=b_{1,0}\left(1-p^{2 M}\right) /(1-p)$, we obtain

$$
\begin{aligned}
& 1=\sum_{i=1}^{2 M} \sum_{k=0}^{W_{\max }^{-1}} b_{i, k} \\
&=\frac{b_{1,0}}{2}[\left(W_{\max }-1\right)\left(1-p^{2 M}\right) \\
&+\left(\frac{5+6 C-W_{\max }}{4}\right) \frac{p^{2}-p^{2 M+2}}{1-p^{2}} \\
&\left.+\left(W_{\max }-1\right) \frac{p^{3}-p^{2 M+1}}{1-p^{2}}\right] .
\end{aligned}
$$

By solving $b_{1,0}, \tau$ is the sum of $b_{i, 0} \forall i \in[1,2 M]$ and is given as $b_{1,0} / 1-p$, which can be represented by $p, W_{\max }$, and $M$.

Next, we calculate $p$. Let the steady-state probabilities of the nodal state Markov chain be denoted as $\pi_{i}, \pi_{s}, \pi_{r}, \pi_{f}$, $\pi_{d}$, and $\pi_{o}$, and let the average time periods that a node stays in the corresponding states be $T_{i}, T_{s}, T_{r}, T_{f}, T_{d}$, and $T_{o}$, respectively. We create a continuous-time state transition process $X=\left\{X_{t}, t \geq 0\right\}$ by defining the node state variable at time $t, X_{t}$, which denotes the state into which the system transitioned at the last transition time occurring before time $t$. Let $\pi^{\prime}$ represent the percentage of time that the node resides in the idle state, i.e.,

$$
\pi^{\prime}=\frac{\pi_{i} T_{i}}{\pi_{i} T_{i}+\pi_{s} T_{s}+\pi_{r} T_{r}+\pi_{f} T_{f}+\pi_{d} T_{d}+\pi_{o} T_{o}} .
$$

Consider a node $S$, whose next packet is to be forwarded to a neighbor node $R$. Then, the probability of failure for $S$ 's packet at an arbitrary time $t_{0}$ is given by

$$
\begin{aligned}
p & =1-\operatorname{Pr}\{\text { success } \mid \text { a transmission is attempted }\} \\
& =1-p_{1} p_{2} p_{3}
\end{aligned}
$$

where

$$
\begin{gathered}
p_{1}=\operatorname{Pr}\left\{\text { receiver node is idle at } t_{0}\right\}=\pi^{\prime} \\
p_{2}=\operatorname{Pr}\{\text { sender's signal strong enough at receiver }\} \\
p_{3}=\operatorname{Pr}\{\text { no stations in sender's beam initiate a } \\
\text { transmission in receiver's direction in } \\
\left.2 t_{r t s}+2 \text { slot times }\right\} .
\end{gathered}
$$

We do not need to consider the probability of the receiver pointing in the direction of the sender when calculating $p_{1}$, since it is assumed that the sender will always capture the receiver by sending $2 M$ DRTS messages. For simplicity, we assume that $p_{2}=1$. $p_{3}$ is given as

$$
\begin{aligned}
& p_{3}=(1-\operatorname{Pr}\{\text { a station is in the sender's beam }\} \\
& \times \operatorname{Pr}\{\text { it transmits }\} \\
& \times \operatorname{Pr}\{\text { its transmission is in } \\
&\text { the direction of the receiver }\})^{(N-2)\left(2 t_{r t s}+2\right)}
\end{aligned}
$$

$$
=\left(1-\pi^{\prime} \tau\left(\frac{1}{M}\right)^{2}\right)^{(N-2)\left(2 t_{r t s}+2\right)} \text {. }
$$


Next, we need to derive the transition and steady-state probabilities. Using (10) and (11), we obtain the transition probabilities as

$$
\begin{aligned}
P_{i s}= & P_{i r}=\tau(1-p) \\
P_{i i}= & (1-\tau) \operatorname{Pr}\{\text { no stations start to transmit } \\
& \quad \text { DRTS or DCTS in its direction }\} \\
= & (1-\tau)\left(1-\pi^{\prime} \tau\left(\frac{1}{M}\right)-\pi^{\prime} P_{i r}\left(\frac{1}{M}\right)\right)^{N-1} \\
P_{i f}=\tau p & \\
P_{s i}= & P_{r i}=P_{f i}=P_{d i}=P_{o i}=1 .
\end{aligned}
$$

The calculation of $P_{i d}$ and $P_{i o}$ is more involved. To simplify the calculation, we use the fact that the ratio of the number of packets per packet type RTS:CTS:DATA:ACK is approximately $M:(1-p):(1-p):(1-p)$. Then, we can write $P_{i d}$ as

$$
\begin{aligned}
& P_{i d}= \operatorname{Pr}\{\text { sender successfully receives an incoming } \\
&\text { packet that is not intended for it }\} \\
& \operatorname{Pr}\{\text { the incoming packet is DRTS/DCTS }\} \\
& \operatorname{Pr}\{\text { it is oriented to the direction } \\
&\quad \text { of the next packet }\} \\
& \approx \\
&\left(1-P_{i i}-P_{i s}-P_{i r}-P_{i f}\right) p_{3} \\
& \times[M+(1-p)] /\left[M^{2}+3 M(1-p)\right]
\end{aligned}
$$

and $P_{i o}$ can then be easily obtained as

$$
P_{i o}=1-P_{i i}-P_{i s}-P_{i r}-P_{i f}-P_{i d} .
$$

By solving the balance equations for the steady-state probabilities, we can obtain

$$
\begin{aligned}
& \pi_{i}=1 /\left(2-P_{i i}\right) \\
& \pi_{s}=P_{i s} \pi_{i}=\pi_{r} \\
& \pi_{f}=P_{i f} \pi_{i} \\
& \pi_{d}=P_{i d} \pi_{i} \\
& \pi_{o}=P_{i o} \pi_{i} .
\end{aligned}
$$

The corresponding time intervals that a station stays in the idle state are given as $T_{i}=\alpha$, where $\alpha$ denotes a BO slot time duration. The average time of a node in the success state is

$$
\begin{aligned}
E\left[T_{s}\right] \approx M & \times \mathrm{DRTS}+M \times \mathrm{SIFS}+\mathrm{DCTS}+\mathrm{SIFS} \\
& +\mathrm{DATA}+\mathrm{SIFS}+\mathrm{ACK}+\mathrm{DIFS}+E\left[\mathrm{BO}_{s}\right]
\end{aligned}
$$

where $E\left[\mathrm{BO}_{s}\right]$ denotes the average $\mathrm{BO}$ spent in a successful transmission, i.e.,

$$
\begin{aligned}
E\left[\mathrm{BO}_{s}\right]=\sum_{i=0}^{2 M-1} E[\mathrm{BO}(i) \mid(\mathrm{i} & +1) \text { DRTS Tx }] \\
& \times \operatorname{Pr}\{(\mathrm{i}+1) \text { DRTS Tx }\} .
\end{aligned}
$$

Assuming that the receiver captures the $i$ th DRTS message with probability $(2 M)^{-1}$ (i.e., with a uniform distribution),
$E\left[\mathrm{BO}_{s}\right]$ is given by

$$
E\left[\mathrm{BO}_{s}\right] \approx\left[5 W_{\max }-2(\mathrm{DRTS}-\mathrm{SIFS})\right] / 16 .
$$

The time spent in the receive state $T_{r}$ is DRTS + SIFS + DCTS + SIFS + DATA + SIFS + ACK + DIFS.

The average time spent in the fail state $E\left[T_{f}\right]$ is approximately $2 M \times($ DRTS + DIFS $)+E\left[\mathrm{BO}_{f}\right]$, where

$$
E\left[\mathrm{BO}_{f}\right] \approx M\left[5 W_{\max }-2(\mathrm{DRTS}-\mathrm{SIFS})\right] / 8 .
$$

The time spent in the defer state $T_{d}$ is equal to the time spent in the success state, i.e., $T_{d}=T_{s}$.

The length of the overhear duration is given by

$$
\begin{aligned}
T_{O}=\text { DIFS }+ & \frac{M \cdot \text { DRTS }}{M+3-3 p} \\
& +\frac{(1-p)(\text { DCTS }+H+\text { DATA }+ \text { ACK })}{M+3-3 p} .
\end{aligned}
$$

The antenna switching time is assumed to be small enough and is ignored in all of our calculations.

An iterative method is used to calculate the optimal $\tau, p$, and $\pi^{\prime}$. Then, the saturation throughput (in bits per second) of a network with $N$ active nodes is calculated as

$$
T H=\frac{N \pi_{s} E[P]}{\pi_{i} T_{i}+\pi_{s} E\left[T_{s}\right]+\pi_{r} T_{r}+\pi_{f} E\left[T_{f}\right]+\pi_{d} T_{d}+\pi_{o} T_{o}}
$$

where $E[P]$ is the average payload size of a data packet.

In the next section, we present our simulation results to validate the analytical model. We investigate the performance of DtD MAC in terms of saturation throughput and fairness. Then, we also present the performance of the DtD MAC protocol when the emerging very high data rate mmWave technology is used.

\section{Performance Evaluation}

\section{A. Simulation Parameters}

We implemented the DtD MAC protocol in QualNet version 4.0 [18]. On top of the 802.11b physical layer model, we implemented the directional transmission and receiving procedures. The values of the parameters used in the simulations are listed in Table I, unless explicitly stated otherwise. The network topology is randomly generated by QualNet. Fourteen nodes are uniformly distributed in a $200 \times 200 \mathrm{~m}^{2}$ area, and they are grouped as seven source-destination pairs. The source-destination pairs are one-hop neighbors that are randomly chosen by the simulator. Each pair is loaded with a constant-bit-rate flow. Each simulation runs for $100 \mathrm{~s}$, and the results are the average of five runs with different initial random seeds. The $95 \%$ confidence intervals of simulation results are also plotted in the figures.

\section{B. Network Throughput}

Fig. 5 compares the throughput obtained from the simulation. For $M=1$, the original 802.11 DCF MAC is used, and 
TABLE I

SIMULATION PARAMETERS

\begin{tabular}{|c|c||c|c|}
\hline Parameter & Value & Parameter & Value \\
\hline \hline Tx power & $15 \mathrm{dBm}$ & Data rate & $2 \mathrm{Mbps}$ \\
Rx threshold & $-81 \mathrm{dBm}$ & Base rate & $1 \mathrm{Mbps}$ \\
Sensing threshold & $-91 \mathrm{dBm}$ & Packet size & $512 \mathrm{Bytes}$ \\
$W_{\max }$ & 64 & Slot time $\sigma$ & $20 \mu \mathrm{S}$ \\
Tx range & $200 \mathrm{~m}$ & SIFS & $10 \mu \mathrm{S}$ \\
DRTS & $160 \mathrm{bits}$ & MAC header & $28 \mathrm{Bytes}$ \\
DCTS/ACK & $112 \mathrm{bits}$ & PHY header & $192 \mathrm{bits}$ \\
\hline
\end{tabular}

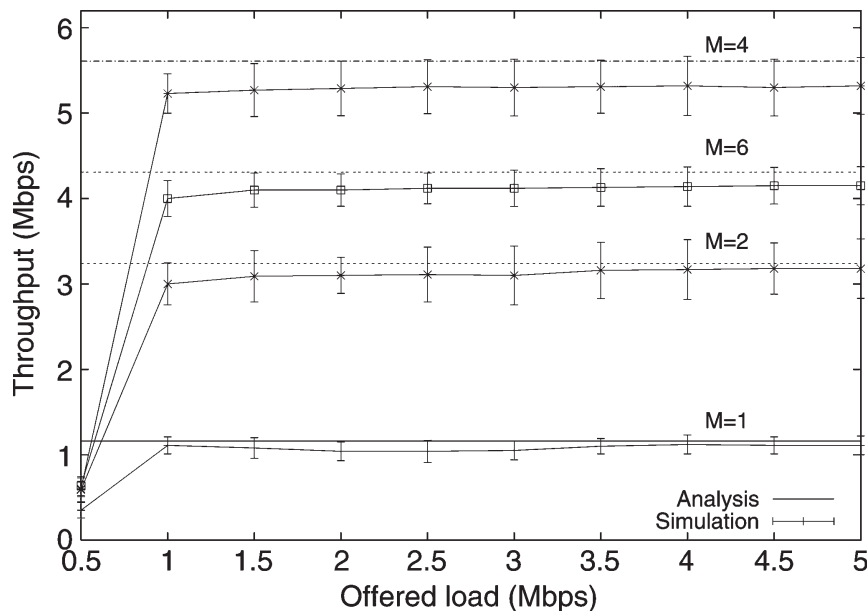

Fig. 5. Total network throughput with offered load for each node pair.

for $M>1$, the proposed DtD MAC protocol is used. The relatively small value of $W_{\max }=64$ is chosen here because of the following: 1) The number of nodes competing in the same direction using directional antennas is lower than that using omnidirectional antennas, and 2) a large number of DRTS messages are missed due to deafness and not collision. The general principle is that $W_{\max }$ could be smaller if $M$ is larger. We observe that, as the offered load increases, the network approaches its maximum saturation throughput. We also observe that the directional antenna cases $(M=2,4$, and 6 ) achieve a higher throughput than the omnidirectional antenna case $(M=1)$, and $M=4$ achieves the highest throughput.

In Fig. 6, we plot the network throughput for the increasing number of antenna sectors, and $W_{\max }$ is chosen to be 64 and 128 , respectively. From the figure, we observe that for a smaller $W_{\max }$, the throughput is higher overall. Furthermore, we can see that when the number of sectors is increased from 2 to 4 , the network throughput is also increased. However, when the number of antenna sectors is further increased to five or six, the throughput is lower than the case when four antenna sectors are used. This is mainly due to the increased overhead of control messages. This leads to the conclusion that there is an optimal number of antenna sectors and $W_{\max }$ for a specific network density. A similar trend was also observed in [19].

Furthermore, Fig. 6 shows the throughput when the directional-to-omnidirectional (DtO) protocol is used [21]. When the number of sectors at the sender is small (i.e., $M=2$ ), we observe a small improvement in throughput compared with the omnidirectional-to-omnidirectional (i.e., $M=1$ ) case. As $M$ further increases, we see an increase in throughput; however, the DtD MAC protocol continues to outperform the DtO MAC

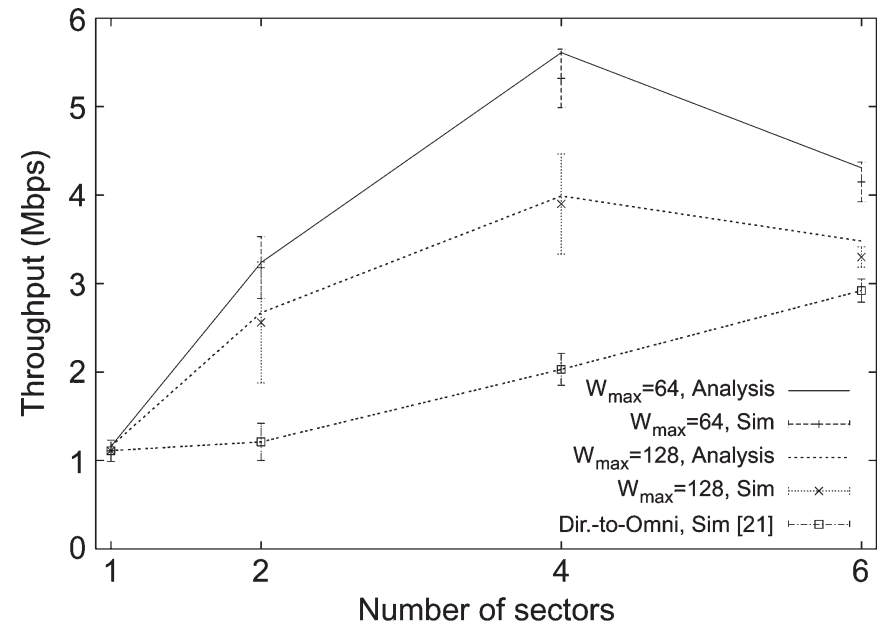

Fig. 6. Network throughput with different number of antenna sectors.

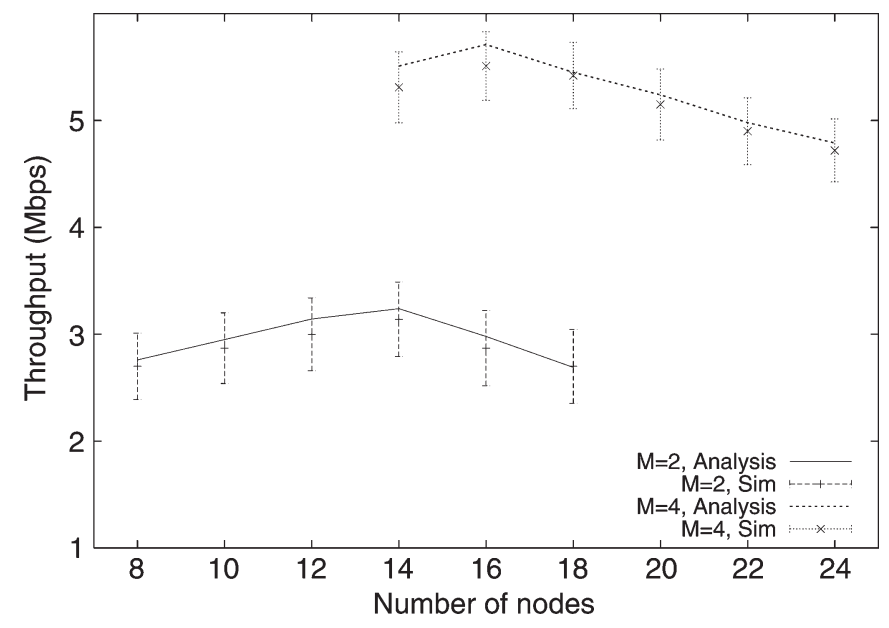

Fig. 7. Network throughput with different number of nodes.

protocol. When $M$ is greater than 4 , we observe a decrease in network throughput for DtD MAC, and the improvement over the DtO MAC shrinks. This, however, is not the case when the network becomes more dense. In networks where the density is higher, the DtD MAC protocol considerably outperforms the DtO MAC protocol. This can intuitively be explained by the fact that in more dense networks, the spatial reuse of the DtD MAC protocol improves, and a greater number of nodes can communicate simultaneously. To further elaborate on this point, Fig. 7 plots the network throughput versus the number of nodes in the network.

Fig. 7 shows that the saturation throughput for a certain number of sectors is maximized at a specific number of nodes. Interestingly, we observe that for a higher number of antenna sectors, the number of nodes at which the throughput is maximized is higher (i.e., 14 versus 16 nodes for two versus four sectors, respectively). This confirms our previous claim, which suggests that a higher number of antenna sectors is desirable for more dense networks.

Fig. 8 shows the effect of packet size on network throughput in the DtD MAC protocol. We can observe an expected trend: As the packet size increases, the throughput increases. This is due to the fact that as the payload size increases and the 


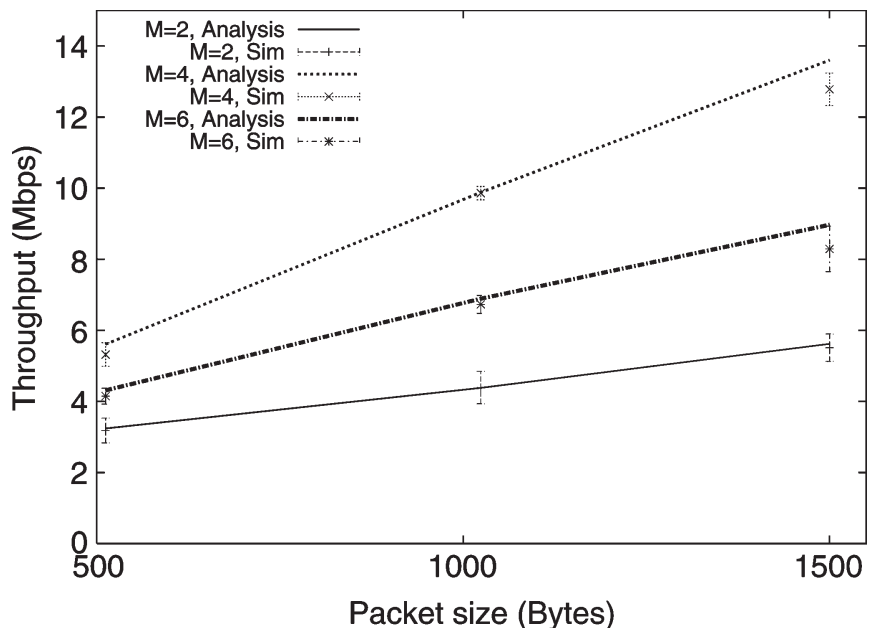

Fig. 8. Network throughput with different packet size.

time needed to transmit the control messages remains the same, the efficiency of the protocol increases. Because the number of nodes used here is 14 , we see that the best performance is achieved in the $M=4$ case. For a more dense network, we expect the throughput to peak at a higher number of antenna sectors. In all cases, the simulation results agree with the analytical results obtained.

\section{Fairness of the DtD MAC Protocol}

To measure the fairness, we use Jain's fairness index [22]. The index uses the throughput of a given node within a group $\left(x_{1}, x_{2}, \ldots, x_{i}\right)$ as a fairness measure. Therefore, the fairness index is defined as

$$
f\left(x_{1}, x_{2}, \ldots, x_{i}\right)=\frac{\left(\sum_{i=1}^{n} x_{i}\right)^{2}}{n \sum_{i=1}^{n} x_{i}^{2}} .
$$

The values of the fairness index are between $1 / n$ and 1 , where 1 indicates the ideal fairness, and $1 / n$ indicates that the fairness is skewed toward one specific node. Using a random topology, we calculate the fairness index when the network is saturated. The results are plotted in Fig. 9. For comparison, we calculated the fairness index when using omnidirectional antennas (i.e., $M=1$ ) and show that DtD MAC can outperform the case with omnidirectional antennas. As the offered load increases and the network becomes more congested, the fairness of the omnidirectional antenna case significantly decreases, whereas DtD MAC continues to achieve a high level of fairness. This is because DtD MAC uses directional DRTS and DCTS control messages and reserves smaller communication areas than using omnidirectional RTS/CTS control messages so that the competition level is reduced, and the collision and starvation problems are alleviated.

\section{DtD MAC Protocol for the mmWave Technology}

In this section, we study the performance of the DtD MAC protocol for the very high data rate mmWave technology. The base rate is $50 \mathrm{Mb} / \mathrm{s}$, and the data rate is $1 \mathrm{~Gb} / \mathrm{s}$ [23]. Due to the limitations of the simulator that does not support mmWave

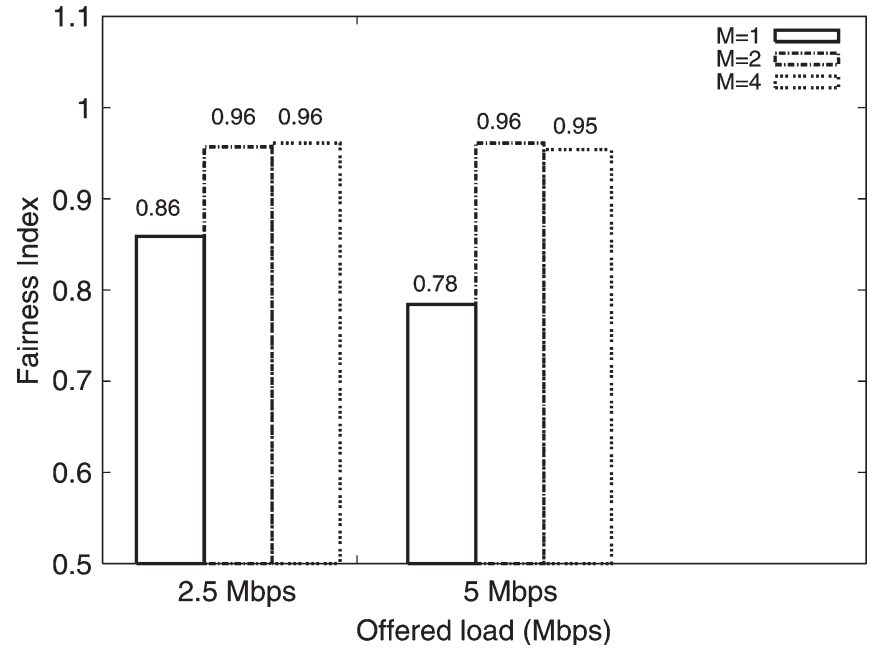

Fig. 9. Fairness index in saturation condition with varying $M$.

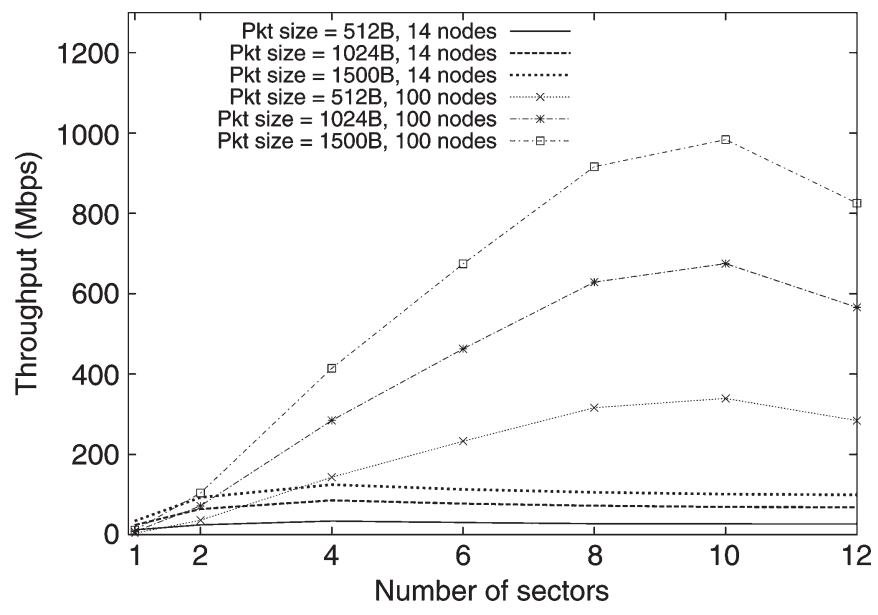

Fig. 10. Millimeter-wave network throughput with different number of nodes and packet size.

transmissions, we only present analytical results here. Fig. 10 shows the saturation throughput of a 14-node network using 512-, 1024-, and 1500-B packets. It can be observed that as the number of sectors increases, the throughput increases (due to a higher spatial reuse and a lower collision probability) and saturates. Then, it begins to slightly decrease. Once again, this can be explained by the increasing overhead when $M$ is large. Because of the high data rates that can be achieved by the mmWave technology, a more interesting and practical case to study is highly dense networks. To consider this case, the protocol performance when there are 100 nodes is plotted in the same figure. We find an interesting phenomenon. Not only is the throughput greater when more nodes are present, but in addition, the trend observed in the case with omnidirectional antennas is reversed. As observed from the bottom left corner of Fig. 10, when the number of nodes is increased, the throughput of the omnidirectional case is decreased; however, the throughput of the DtD MAC protocol is increased. This is due to the increase in spatial reuse that is achieved by using directional antennas. This observation leads to the conclusion that the DtD MAC protocol is indeed desirable for highly dense 


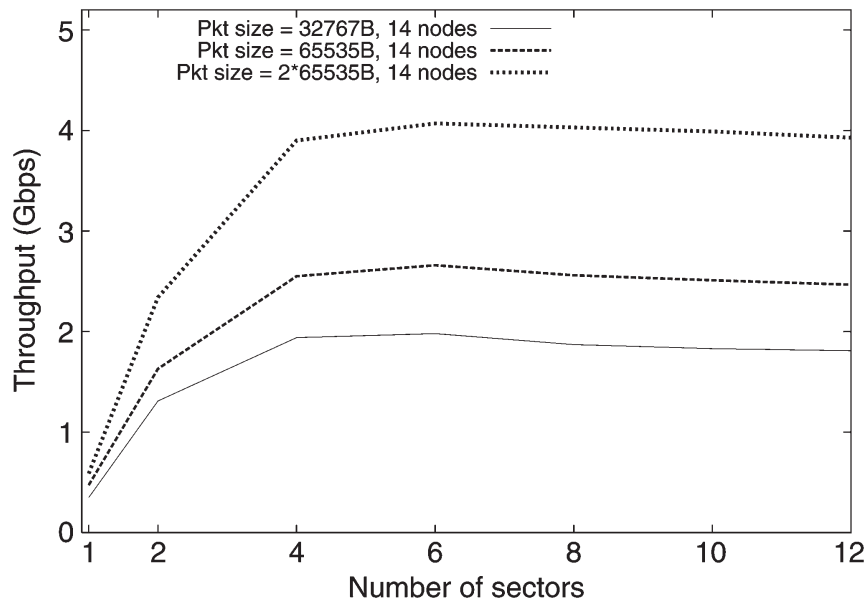

Fig. 11. Millimeter-wave network throughput with extended packet sizes.

networks where the physical layer data rates are very high (e.g., mmWave).

From Fig. 10, the saturation throughput is quite low (considering the $1-\mathrm{Gb} / \mathrm{s}$ data rate and $50-\mathrm{Mb} / \mathrm{s}$ base rate) due to the PHY/MAC layer overheads. On the other hand, the IEEE 802.15.3c (mmWave) standard [23] allows the maximum payload size of $65535 \mathrm{~B}$. Therefore, we plot the results for packet sizes of 32767,65535 , and $2 \times 65535$ B, respectively. From Fig. 11, the network throughput using these extended packet sizes is in the range of $0.5-4 \mathrm{~Gb} / \mathrm{s}$. This shows that the DtD MAC protocol performs well when large payload sizes are used. This is crucial since for high-data-rate technologies, large payload sizes are often used due to the big difference between the base and data rates.

\section{RELATED WORK}

Nasipuri et al. proposed a MAC protocol that uses a variation of the IEEE 802.11 DCF to support nodes with directional antennas [6]. In their protocol, the RTS and CTS messages are omnidirectionally sent, and then, the DATA and ACK packets are sent directionally. Building upon this, Ko et al. proposed a MAC protocol that sends a DRTS when at least one of the antenna beams is blocked, or an omnidirectional RTS otherwise, followed by an omnidirectional CTS from the receiver [7]. It was assumed that all nodes know the locations of their neighbors. To explore the advantage when multiple hops may be available, Choudhury et al. proposed a multihop RTS directional MAC [10], [24]. In their protocol, a sending node propagates the RTS to the destination over multiple intermediate nodes that omnidirectionally listen, and the CTS, DATA, and ACK messages are directionally exchanged. The use of both directional and omnidirectional modes introduces the asymmetry-in-gain problem. In this paper, we address this issue by using directional antennas only.

Wang et al. [25] proposed a directional MAC protocol for ad hoc networks that assumes full network-wide synchronization, which is called Synchronized Directional MAC protocol. Multiple node pairs contend for the channel in a random-access phase. Parallel collision-free DATA and ACK are sent during the DATA and ACK phases, respectively. However, achieving network-wide synchronization is difficult in general and can significantly increase the cost of deployment. For this reason, we designed the DtD MAC protocol to work asynchronously.

The work of Singh and Singh [26] and Huang et al. [27] proposed busy tone-based directional MAC protocols. The protocols call for idle nodes to listen in the omnidirectional mode. In case a node receives a sender busy tone, it forms a beam toward the sender and transmits a receiver tone. DATA and ACK are then exchanged. As busy tone protocols often require two channels to operate, they may not be preferable due to cost or bandwidth limitations. In addition, if multiple nodes simultaneously transmit the busy tone, then spatial reuse may be reduced. The DtD MAC protocol does not use busy tones, i.e., control and data packets are sent over the same channel.

Takai et al. [8] proposed directional virtual carrier sensing (DVCS), which is a mechanism that consists of using DNAV, AoA, and beam locking and unlocking. DVCS can be used to enhance the performance of directional MAC protocols. Hsu and Rubin [21] proposed an analytical model to study the throughput performance of directional carrier sense multiple access with collision-avoidance MAC protocols. The proposed model assumed directional transmission and omnidirectional reception. The DtD MAC protocol adopts the DNAV and AoA caching techniques from DVCS as well, and we extended the analytical work in [21] to study the performance of the DtD protocol.

For directional-antenna-only networks, Zhang et al. [28] proposed a time-division-multiple-access-based MAC protocol called Link ScheduLer for Directional Antennas (LiSL/d). Jakllari et al. proposed a MAC protocol called Polling-based MAC protocol (PMAC) [29]. With PMAC, a node polls its one-hop neighbors to obtain their location information and schedules transmissions and receptions. Although LiSL/d and PMAC eliminate the asymmetry-in-gain problem, they require network synchronization. Furthermore, the optimal frame duration is a system parameter that may be difficult to obtain in dynamic network conditions, and the polling scheme is not efficient if the traffic is bursty. To the best of our knowledge, none of the directional-antenna-only protocols proposed thus far operate in a distributed and asynchronous manner. These reasons motivated the development of the DtD MAC protocol.

\section{CONCLUSiOn AND Future WORK}

In this paper, we have outlined the design of the DtD MAC protocol that supports DtD transmissions. The DtD MAC protocol is fully distributed, does not require synchronization, eliminates the asymmetry-in-gain problem, and alleviates the effect of deafness problems in directional-antenna-only networks. The performance of the protocol has been studied through an analytical model and verified by simulation. The analytical and simulation results show that DtD MAC can considerably improve the network throughput and fairness. The feasibility of DtD MAC has also been studied for the very high data rate mmWave technology, and the results are promising. It has been shown that as the number of nodes in a network increases, the DtD MAC protocol can significantly improve the 
network throughput when the number of directional antenna sectors is properly chosen.

There are many related research issues that beckon further investigation: 1) how to quantify the protocol performance and optimize the system parameters (such as $M$ and packet size) considering user mobility; 2) how different wireless channel error profiles affect the performance of the DtD MAC; and 3) how to quantify the protocol performance in a multihop wireless networks. These issues constitute our ongoing and future research efforts.

\section{REFERENCES}

[1] Y. Wang and J. J. Garcia-Luna-Aceves, "Spatial reuse and collision avoidance in ad hoc networks with directional antennas," in Proc. GLOBECOM, Nov. 2002, pp. 112-116.

[2] T. Issariyakul, H. Ekram, and K. Dong, "Medium access control protocols for wireless mobile ad hoc networks: Issues and approaches," Wirel. Commun. Mob. Comput., vol. 3, no. 8, pp. 935-958, Dec. 2003.

[3] IEEE 802.15.3c Task Group. [Online]. Available. http://www.ieee802. org/15/pub/TG3c.html

[4] S. Yong and C. Chong, "An overview of multigigabit wireless through millimeter wave technology: Potentials and technical challenges," EURASIP J. Wireless Commun. Netw., vol. 2007, no. 1, p. 50, Jan. 2007.

[5] L. X. Cai, L. Cai, X. Shen, and J. Mark, "Efficient resource management for mmWave WPANs," in Proc. WCNC, Mar. 2007, pp. 3816-3821.

[6] A. Nasipuri, S. Ye, J. You, and R. E. Hiromoto, "A MAC protocol for mobile ad hoc networks using directional antennas," in Proc. WCNC, Sep. 2000, pp. 1214-1219.

[7] Y. Ko, V. Shankarkumar, and N. Vaidya, "Medium access control protocols using directional antennas in ad hoc networks," in Proc. INFOCOM, Mar. 2000, pp. 13-21.

[8] M. Takai, J. Martin, A. Ren, and R. Bagrodia, "Directional virtual carrier sensing for directional antennas in mobile ad hoc networks," in Proc. MobiHoc, Jun. 2002, pp. 183-193.

[9] R. Ramanathan, J. Redi, C. Santivanez, D. Wiggins, and S. Polit, "Ad hoc networking with directional antennas: A complete system solution," IEEE J. Sel. Areas Commun., vol. 23, no. 3, pp. 496-506, Mar. 2005.

[10] R. Choudhury, X. Yang, R. Ramanathan, and N. Vaidya, "Using directional antennas for medium access control in ad hoc networks," in Proc. MOBICOM, Mar. 2002, pp. 59-70.

[11] H. Gossain, C. Cordeiro, D. Cavalcanti, and D. P. Agrawal, "The deafness problems and solutions in wireless ad hoc networks using directional antennas," in Proc. GLOBECOM, Nov./Dec. 2004, pp. 108-113.

[12] G. Jakllari, I. Broustis, T. Korakis, S. V. Krishnamurthy, and L. Tassiulas, "Handling asymmetry in gain in directional antenna equipped ad hoc networks," in Proc. PIMRC, Sep. 2005, pp. 1284-1288.

[13] E. Shihab, "A directional-to-directional mac protocol for ad-hoc networks," M.S. thesis, Univ. Victoria, Victoria, BC, Canada, 2008.

[14] W. X. Sheng, J. Zhou, D. G. Fang, and Y. C. Gu, "Super-resolution DOA estimation in switch beam smart antenna," in Proc. ISAPE, Aug. 2000, pp. 603-606.

[15] N. Karmakar and M. Bialkowski, "Electronically steerable array antennas for mobile satellite communications-A review," in Proc. ICPAST, May 2000, pp. 81-84.

[16] R. Ramanathan, "On the performance of ad hoc networks with beamforming antennas," in Proc. 2nd ACM Int. Symp. MobiHoc, 2001, pp. 95-105.

[17] G. Bianchi, "Performance analysis of the IEEE 802.11 distributed coordination function," IEEE J. Sel. Areas Commun., vol. 18, no. 3, pp. 535-547, Mar. 2000.

[18] Qualnet Version 4.0. [Online]. Available: www.scalable-networks.com

[19] N. Ehsan and R. L. Cruz, "Optimal rate and beam-width control in wireless ad-hoc networks with directional antennas," in Proc. ICC, Jun. 2007, pp. 4943-4948.

[20] E. Shihab, L. Cai, and J. Pan, "A distributed directional-to-directional MAC protocol for asynchronous ad hoc networks," in Proc. GLOBECOM, Nov./Dec. 2008, pp. 4971-4975.

[21] J. Hsu and I. Rubin, "Performance analysis of directional CSMA/CA MAC protocol in mobile ad hoc networks," in Proc. ICC, Jun. 2006, pp. 3657-3662.

[22] R. Jain, The Art of Computer Systems Performance Evaluation. New York: Wiley, 1991
[23] "Merged proposal: New PHY layer and enhancement of MAC for mmWave system proposal," IEEE 802.15 WPAN Millimeter Wave Alternative PHY Task Group 3c (TG3c), Nov. 2007.

[24] R. Choudhury, X. Yang, R. Ramanathan, and N. Vaidya, "On designing MAC protocols for wireless networking using directional antennas," IEEE Trans. Mobile Comput., vol. 5, no. 5, pp. 477-491, May 2006.

[25] J. Wang, Y. Fang, and D. Wu, "SYN-DMAC: A directional MAC protocol for ad hoc networks with synchronization," in Proc. MILCOM, Oct. 2005, pp. 2258-2263.

[26] H. Singh and S. Singh, "Tone based mac protocol for use with adaptive array antennas," in Proc. WCNC, Mar. 2004, pp. 1246-1251.

[27] Z. Huang, C. Shen, C. Srisathapornphat, and C. Jaikaeo, "A busy-tone based directional MAC," in Proc. MILCOM, Oct. 2002, pp. 1233-1238.

[28] Z. Zhang, B. Ryu, G. Nallamothu, and Z. Huang, "Performance of alldirectional transmission and reception algorithms in wireless ad hoc networks with directional antennas," in Proc. MILCOM, Oct. 2005, pp. $225-230$

[29] G. Jakllari, W. Luo, and S. V. Krishnamurthy, "An integrated neighbor discovery and MAC protocol for ad hoc network using directional antennas," IEEE Trans. Wireless Commun., vol. 6, no. 3, pp. 1114-1124, Mar. 2007.

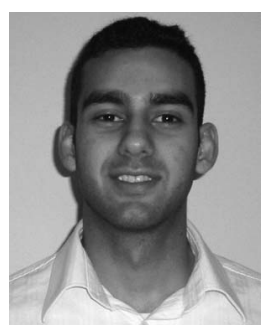

Emad Shihab (S'05) received the B.Eng. and M.A.Sc. degrees in electrical and computer engineering from the University of Victoria, Victoria, BC, Canada, in 2006 and 2008, respectively. He is currently working toward the Ph.D. degree in computer science with Queen's University, Kingston, ON, Canada.

He has held various coop positions with Research In Motion, Waterloo, ON, and Telus Communication Inc., Vancouver, BC. His research spans several areas in wireless communications and networking, with special interest in distribution technologies, network architectures, protocol design, capacity analysis, and resource management of broadband wireless and wired networks.

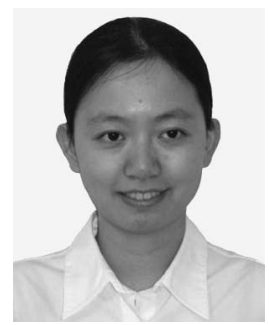

Lin Cai (S'00-M'06) received the M.A.Sc. and $\mathrm{Ph} . \mathrm{D}$. degrees (with Outstanding Achievement in Graduate Studies Award) in electrical and computer engineering from the University of Waterloo, Waterloo, ON, Canada, in 2002 and 2005, respectively.

Since July 2005, she has been an Assistant Professor with the Department of Electrical and Computer Engineering, University of Victoria, BC, Canada. Her research interests span several areas in wireless communications and networking, with a focus on network protocol and architecture design supporting emerging multimedia traffic over wireless, mobile, ad hoc, and sensor networks.

Dr. Cai has served as an Associate Editor for IEEE TRANSACTIONS ON VehICULAR TECHNOLOGY since 2007, the EURASIP Journal on Wireless Communications and Networking since 2006, and the International Journal of Sensor Networks since 2006.

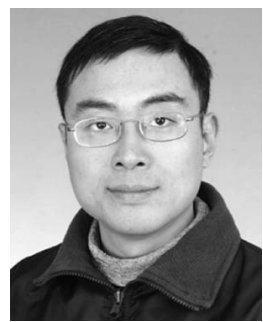

Jianping Pan (S'96-M'98-SM'08) received the B.S. and Ph.D. degrees in computer science from Southeast University, Nanjing, China.

$\mathrm{He}$ did his postdoctoral research with the University of Waterloo, Waterloo, ON, Canada. He also worked at Fujitsu Labs and NTT Labs. He is currently an Assistant Professor of computer science with the University of Victoria, Victoria, BC, Canada. His area of specialization is computer networks and distributed systems, and his recent research interests include protocols for advanced networking, performance analysis of networked systems, and applied network security.

Dr. Pan is a Senior Member of the Association for Computing Machinery. 